\title{
Prognostic factors of postoperative seizure outcomes in older patients with temporal lobe epilepsy
}

\author{
Xinghui He, PhD, ${ }^{1,2}$ Jian Zhou, MD,,2 Yuguang Guan, MD,, Feng Zhai, MD,, ${ }^{1,2}$ Tianfu Li, PhD,, ${ }^{1,3}$ and \\ Guoming Luan, MD, PhD'-3 \\ ${ }^{1}$ Beijing Key Laboratory of Epilepsy, Sanbo Brain Hospital, Capital Medical University; ${ }^{2}$ Department of Neurosurgery, Epilepsy \\ Center, Sanbo Brain Hospital, Capital Medical University; and ${ }^{3}$ Beijing Institute for Brain Disorders, Capital Medical University, \\ Beijing, People's Republic of China
}

OBJECTIVE The authors of this study aimed to investigate surgical outcomes and prognostic factors in older patients with drug-resistant temporal lobe epilepsy (TLE) who had undergone resective surgery.

METHODS Data on patients older than 45 years of age with drug-resistant TLE who had undergone resective surgery at Sanbo Brain Hospital, Capital Medical University, between January 2009 and August 2017 were retrospectively collected. Postoperative seizure outcomes were evaluated according to the International League Against Epilepsy (ILAE) classification. Patients belonging to ILAE classes 1 and 2 were classified as having a favorable outcome, whereas patients belonging to ILAE classes 3-6 were classified as having an unfavorable outcome. Univariate analysis and multivariate logistic regression analysis were used to identify the potential predictors of seizure outcomes.

RESULTS A total of 45 patients older than 45 years of age who had undergone resective epilepsy surgery for TLE were included in the present study. Eight (17.8\%) of 45 patients had preoperative comorbidity in addition to seizures. The average age at the time of surgery was 51.76 years, and the average duration of epilepsy at the time surgery was 18.01 years. After an average follow-up period of $4.53 \pm 2.82$ years (range 2-10 years), $73.3 \%$ (33/45) of patients were seizure free. Surgical complications were observed in $13.3 \%$ of patients. Univariate and multivariate analyses revealed that an MRI-negative finding is the only independent predictor of unfavorable seizure outcomes $(\mathrm{OR} 0.06,95 \% \mathrm{Cl} 0.01-0.67, \mathrm{p}$ $=0.023$ ).

CONCLUSIONS Resective surgery is a safe and effective treatment for older patients with drug-resistant TLE. An MRInegative finding independently predicts unfavorable seizure outcomes.

https://thejns.org/doi/abs/10.3171/2020.1.FOCUS19796

KEYWORDS temporal lobe epilepsy; drug-resistant epilepsy; epilepsy surgery; seizure outcomes

$\mathrm{T}$ EMPORAL lobe epilepsy (TLE) is the most common type of focal drug-resistant epilepsy. ${ }^{10}$ For patients with drug-resistant TLE, complete seizure control cannot be achieved despite the administration of two or more well-tolerated, appropriately chosen antiepileptic drugs (AEDs), whereas resective surgery is an effective therapy in a majority of appropriately selected patients. ${ }^{20}$ Approximately $70 \%$ of patients with TLE show seizure freedom after resective epilepsy surgery. ${ }^{14,18}$ However, most studies reporting the postoperative seizure outcomes in those with TLE have focused on relatively young patients. ${ }^{19}$ Many have considered resective epilepsy surgery to be inappropriate for older patients. First, higher incidences of comorbid medical conditions in older patients can increase the surgical risks. ${ }^{9}$ Second, increased age at surgery and longer seizure duration are associated with poor postoperative seizure outcomes, ${ }^{15}$ which may lead to the expectation that older patients have a decreased chance of experiencing postoperative seizure freedom.

In recent years, some studies have described surgical

ABBREVIATIONS AED = antiepileptic drug; ATL = anterior temporal lobectomy; EEG = electroencephalography; FCD = focal cortical dysplasia; ILAE = International League Against Epilepsy; MEG = magnetoencephalography; TLE = temporal lobe epilepsy.

SUBMITTED October 8, 2019. ACCEPTED January 24, 2020.

INCLUDE WHEN CITING DOI: 10.3171/2020.1.FOCUS19796. 
outcomes in drug-resistant epilepsy patients older than 45 years, ${ }^{1,7,8,13,22,24-26,29}$ but the results have been conflicting. Most studies have shown that resective surgery in older patients has seizure outcomes and complications similar to those in younger patients, ${ }^{1,7,8,22,24-26}$ but again contradictory results have also been reported. Grivas et al. ${ }^{13}$ found that older patients have a higher risk of complications, and Sirven et al. ${ }^{29}$ reported that older patients have a reduced chance of attaining postoperative seizure freedom. Although there has been an increase in the number of peerreviewed case series published in recent years, the study populations have been relatively small, and no study to date has focused on the prognostic factors in older patients with TLE who have undergone resective surgery.

In the present study, we retrospectively investigated postoperative seizure outcomes and analyzed their potential prognostic factors in a case series including 45 older patients with TLE who had undergone resective surgery.

\section{Methods \\ Patients}

This study was approved by the Ethics Committee of Sanbo Brain Hospital, Capital Medical University, and written informed consent was obtained from all patients.

Data on older patients with TLE who had undergone resective surgery at Sanbo Brain Hospital, Capital Medical University, between January 2009 and June 2017 were collected retrospectively. The study inclusion criteria were as follows: 1) patients with drug-resistant TLE who had undergone resective surgery; 2) patients whose age at the time of surgery was 45 years or older; and 3) patients with a follow-up of at least 2 years after surgery. The exclusion criteria were as follows: 1) patients whose age at the time of surgery was younger than 45 years; 2) patients with drug-resistant extratemporal epilepsy; 3) patients who had undergone palliative surgery such as vagus nerve stimulation; and 4) patients with a follow-up of less than 2 years after surgery.

Detailed data on demographic characteristics, clinical variables, and comorbidities that may have influenced surgical outcomes were retrospectively collected from patients' medical records.

\section{Preoperative Evaluation}

Seizure semiology, neurological examination, brain MRI on a 1.5- or 3.0-T unit, and long-term video scalp electroencephalography (EEG) were performed in all patients as standard investigations. If the findings from these studies were not concordant, additional examinations such as PET, magnetoencephalography (MEG), and/or invasive EEG were used to help locate the epileptogenic zones. Surgical decisions were made, and the extent of resection was recommended by neurologists, neurosurgeons, neuroradiologists, and electrophysiologists after detailed preoperative evaluations.

\section{Surgical Procedure}

The purpose of surgical treatment for TLE is to remove the epileptogenic zones and epileptogenic lesions. The epileptogenic zones were defined according to detailed presurgical evaluation and/or intraoperative electrocorticography. Some patients underwent a standard anterior temporal lobectomy (ATL). In the dominant hemisphere, $3.0-4.0 \mathrm{~cm}$ of the anterior lateral temporal lobe was resected. In the nondominant hemisphere, $4.0-5.0 \mathrm{~cm}$ of the anterior lateral temporal lobe was removed. There was no difference in mesial section size between dominant and nondominant hemispheres. Mesial resection included removal of the amygdala and the anterior $3.0 \mathrm{~cm}$ of the hippocampus. Other patients underwent ATL plus lesionectomy.

\section{Surgical Outcomes}

All patients had a follow-up period of at least 2 years after surgery. Postoperative seizure outcomes were assessed according to the International League Against Epilepsy (ILAE) classification. ${ }^{31}$ Favorable outcomes were defined as ILAE classes 1 and 2 during the last 2 years of follow-up, and unfavorable outcomes were defined as ILAE classes 3-6 during the last 2 years of follow-up.

\section{Statistical Analysis}

Continuous variables were described using means \pm standard deviations. Categorical variables were described using frequencies and percentages. In the univariate analysis, continuous variables were cut off according to Youden's index in a receiver operating characteristic curve analysis, and then variables were assessed using Pearson's chi-square or Fisher's exact test. Variables with a $p$ value $<0.1$ in the univariate analysis were entered into a binary logistic regression model in a backward manner. A p value $<0.05$ was considered statistically significant.

\section{Results \\ Patient Characteristics}

A total of 45 patients were included in the present study; 23 were men and 22 were women. The mean age at surgery was $51.76 \pm 6.18$ years (range $45.00-67.00$ years), and the mean age at seizure onset was $33.74 \pm 15.39$ years (range 2.00-63.00 years). The mean duration of seizures by the time of surgery was $18.01 \pm 13.12$ years (range $1.00-53.00$ years), and the mean monthly seizure frequency was $13.77 \pm 24.2$ times. Nine $(20.0 \%)$ patients had partial seizures only, and the remaining $36(80.0 \%)$ had secondary generalized tonic-clonic seizures. Auras were reported in $25(55.6 \%)$ patients. Epileptic risk factors were observed in $5(11.1 \%)$ patients (4 had a history of febrile seizures and 1 had a history of encephalitis). At the time of surgery, 8 (17.8\%) patients had preoperative comorbidities: 5 had hypertension, 2 had diabetes, and 1 had both hypertension and diabetes. Other patient characteristics are shown in Table 1.

\section{Results of Preoperative Evaluation}

Brain MRI results were reviewed in all patients. Temporal lobe lesions were detected in $39(86.7 \%)$ patients, whereas no lesions were detected in the other 6 (13.3\%) patients. During scalp EEG monitoring, interictal epileptic discharges were observed in all patients, and 30 were 
TABLE 1. Demographic and clinical characteristics and their relationship with seizure outcomes in 45 patients

\begin{tabular}{|c|c|c|c|}
\hline Variable & $\begin{array}{c}\text { Favorable } \\
\text { Outcome }\end{array}$ & $\begin{array}{c}\text { Unfavorable } \\
\text { Outcome }\end{array}$ & $p$ Value \\
\hline \multicolumn{4}{|l|}{ Sex } \\
\hline Male & $18(78.26)$ & $5(21.74)$ & \multirow{2}{*}{0.445} \\
\hline Female & $15(68.18)$ & $7(31.82)$ & \\
\hline \multicolumn{4}{|l|}{ Age at surgery } \\
\hline$\leq 52.5 \mathrm{yrs}$ & $18(64.29)$ & $10(35.71)$ & \multirow{2}{*}{$0.096^{*}$} \\
\hline$>52.5 \mathrm{yrs}$ & $15(88.24)$ & $2(11.76)$ & \\
\hline \multicolumn{4}{|l|}{ Seizure duration } \\
\hline$\leq 4.5 \mathrm{yrs}$ & $9(90.00)$ & $1(10.00)$ & \multirow{2}{*}{$0.246^{*}$} \\
\hline$>4.5 \mathrm{yrs}$ & $24(68.57)$ & $11(31.43)$ & \\
\hline \multicolumn{4}{|l|}{ Age at seizure onset } \\
\hline$\leq 41 \mathrm{yrs}$ & $19(65.52)$ & $10(34.48)$ & \multirow{2}{*}{$0.164^{*}$} \\
\hline$>41 \mathrm{yrs}$ & $14(87.50)$ & $2(12.50)$ & \\
\hline \multicolumn{4}{|l|}{ Monthly seizure frequency } \\
\hline$\leq 7$ times & $25(80.65)$ & $6(19.35)$ & \multirow{2}{*}{0.099} \\
\hline$>7$ times & $8(57.14)$ & $6(42.86)$ & \\
\hline \multicolumn{4}{|l|}{ Seizure types } \\
\hline Partial & $6(66.67)$ & $3(33.33)$ & \multirow{2}{*}{$0.682^{*}$} \\
\hline sGTCS & $27(75.00)$ & $9(25.00)$ & \\
\hline \multicolumn{4}{|l|}{ Auras } \\
\hline Yes & $18(72.00)$ & $7(28.00)$ & \multirow{2}{*}{0.821} \\
\hline No & $15(75.00)$ & $5(25.00)$ & \\
\hline \multicolumn{4}{|l|}{ Epileptic risk factors } \\
\hline Yes & $5(100.00)$ & $0(0.00)$ & \multirow{2}{*}{$0.303^{*}$} \\
\hline No & $28(70.00)$ & $12(30.00)$ & \\
\hline \multicolumn{4}{|l|}{ Comorbidities } \\
\hline Yes & $7(87.50)$ & $1(12.50)$ & \multirow{2}{*}{$0.419^{*}$} \\
\hline No & $26(70.27)$ & $11(29.73)$ & \\
\hline \multicolumn{4}{|l|}{ Side of surgery } \\
\hline Rt & $13(72.22)$ & $5(27.78)$ & \multirow{2}{*}{0.891} \\
\hline $\mathrm{Lt}$ & $20(74.07)$ & $7(25.93)$ & \\
\hline \multicolumn{4}{|l|}{ Surgical type } \\
\hline ATL & $20(68.97)$ & $9(31.03)$ & \multirow{2}{*}{$0.491^{*}$} \\
\hline ATL plus lesionectomy & $13(81.25)$ & $3(18.75)$ & \\
\hline \multicolumn{4}{|l|}{ MRI result } \\
\hline Positive & $31(79.49)$ & $8(20.51)$ & \multirow{2}{*}{$0.035^{*} \dagger$} \\
\hline Negative & $2(33.33)$ & $4(66.67)$ & \\
\hline PET performed & & & \\
\hline Yes & $9(64.29)$ & $5(35.71)$ & 0356 \\
\hline No & $24(77.42)$ & $7(22.58)$ & 0.350 \\
\hline MEG performed & & & \\
\hline Yes & $12(70.59)$ & $5(29.41)$ & 0746 \\
\hline No & $21(75.00)$ & $7(25.00)$ & 0.140 \\
\hline IEDs & & & \\
\hline Unilat & $24(80.00)$ & $6(20.00)$ & 0.153 \\
\hline Bilat & $9(60.00)$ & $6(40.00)$ & 0.150 \\
\hline
\end{tabular}

» CONTINUED FROM PREVIOUS COLUMN

TABLE 1. Demographic and clinical characteristics and their relationship with seizure outcomes in 45 patients

\begin{tabular}{|c|c|c|c|}
\hline Variable & $\begin{array}{l}\text { Favorable } \\
\text { Outcome }\end{array}$ & $\begin{array}{l}\text { Unfavorable } \\
\text { Outcome }\end{array}$ & $\mathrm{p}$ Value \\
\hline \multicolumn{4}{|l|}{ Ictal onset rhythms } \\
\hline Unilat & $14(70.00)$ & $6(30.00)$ & \multirow{3}{*}{0.540} \\
\hline Bilat & $8(66.67)$ & $4(33.33)$ & \\
\hline Unknown & $11(84.62)$ & $2(15.38)$ & \\
\hline \multicolumn{4}{|l|}{ Invasive EEG } \\
\hline Yes & $3(50.00)$ & $3(50.00)$ & \multirow{2}{*}{$0.319^{*}$} \\
\hline No & $30(76.92)$ & $9(23.08)$ & \\
\hline \multicolumn{4}{|l|}{ Neuropathology } \\
\hline Hippocampal sclerosis & $16(80.00)$ & $4(20.00)$ & \multirow{5}{*}{0.180} \\
\hline Vascular malformations & $7(100.00)$ & $0(0.00)$ & \\
\hline FCD type IB & $3(50.00)$ & $3(50.00)$ & \\
\hline Tumors & $5(62.50)$ & $3(37.50)$ & \\
\hline Other & $2(50.00)$ & $2(50.00)$ & \\
\hline \multicolumn{4}{|l|}{ Surgical complications } \\
\hline Yes & $5(83.33)$ & $1(16.67)$ & \multirow{2}{*}{$0.99^{*}$} \\
\hline No & $28(71.79)$ & $11(28.21)$ & \\
\hline \multicolumn{4}{|l|}{ Acute postop seizuresł } \\
\hline Yes & $2(66.67)$ & $1(33.33)$ & \multirow{2}{*}{$0.99^{*}$} \\
\hline No & $31(73.81)$ & $11(26.19)$ & \\
\hline
\end{tabular}

IED = interictal epileptic discharge; sGTCS = secondary generalized tonicclonic seizure.

Values are presented as the number of patients (\%) unless otherwise indicated.

* For comparisons of binary variables, Fisher's exact test was used.

$+p<0.05$.

$\ddagger$ Seizures occurred during the 1st week after surgery.

unilateral and 15 were bilateral. Seizures were recorded in $32(71.1 \%)$ patients, $20(44.4 \%)$ of whom had unilateral epileptic discharges and $12(26.7 \%)$ of whom had bilateral epileptic discharges. Seizures failed to record in 13 (28.9\%) patients during the scalp EEG recording. In addition, to help locate the epileptogenic focus, PET was performed in $14(31.1 \%)$ patients, MEG was performed in 17 $(37.8 \%)$ patients, and invasive EEG was performed in 6 (13.3\%) patients.

\section{Surgical Outcomes and Complications}

Twenty-nine patients underwent a standard ATL, and 16 underwent ATL plus lesionectomy. After a follow-up period of $2-10$ years (mean $4.53 \pm 2.82$ years), 33 (73.3\%) patients were seizure free, with $32(71.1 \%)$ patients showing ILAE class 1 outcomes and $1(2.2 \%)$ patient having an ILAE class 2 outcome. Of the other 12 (26.7\%) patients, 4 (8.9\%) belonged to ILAE class 3, $6(13.3 \%)$ to ILAE class 4 , and 2 (4.4\%) to ILAE class 6; none of the patients had an ILAE class 5 outcome.

There were no operative or perioperative deaths. Surgical complications were observed in $6(13.3 \%)$ patients; 3 (6.7\%) had aphasia and $3(6.7 \%)$ had mild hemiparesis, 5 
TABLE 2. Predictors of seizure outcomes after resective surgery for older patients with TLE, on multivariate analysis

\begin{tabular}{lccc}
\hline \multicolumn{1}{c}{ Variable } & OR & $95 \% \mathrm{Cl}$ & $\mathrm{p}$ Value \\
\hline Age at surgery $\leq 52.5$ yrs & 8.75 & $0.93-81.91$ & 0.057 \\
\hline Monthly seizure frequency $\leq 7$ times & 0.43 & $0.09-2.06$ & 0.292 \\
\hline MRI result (positive) & 0.06 & $0.01-0.67$ & $0.023^{*}$ \\
\hline
\end{tabular}

${ }^{*} p<0.05$.

$(11.1 \%)$ of whom recovered completely within 1 year after surgery. One (2.2\%) patient had permanent mild aphasia. No wound infections or postoperative intracerebral hemorrhages were observed in the patient cohort. Quadrantanopia was not considered a surgical complication in the present study.

\section{Neuropathology}

The resected tissues were processed for routine histological analysis. Hippocampal sclerosis was observed in $20(44.4 \%)$ patients, and focal cortical dysplasia (FCD) type IB was diagnosed in $6(13.3 \%)$ patients, vascular malformations in $7(15.6 \%)$ patients (6 cavernous malformations and 1 arteriovenous malformation), tumors in 8 (17.8\%) patients (5 gangliogliomas, 2 dysembryoplastic neuroepithelial tumors, and 1 astrocytoma WHO grade II), a glial scar in $1(2.2 \%)$ patient, heterotopia in $1(2.2 \%)$ patient, nonspecific gliosis in $1(2.2 \%)$ patient, and an epidermoid cyst in $1(2.2 \%)$ patient.

\section{Predictors of Seizure Outcomes}

In the univariate analysis, the preoperative MRI result was the only factor associated with postoperative seizure outcome (Table 1). For the multivariate analysis, the variables, including age at surgery, monthly seizure frequency, and MRI results, that showed a $\mathrm{p}$ value $<0.1$ on univariate analysis were entered into a binary logistic regression model in a backward manner. Multivariate analysis revealed that a negative preoperative MRI finding was the only independent predictor of an unfavorable seizure outcome (OR 0.06, 95\% CI 0.01-0.67, p = 0.023; Table 2).

\section{Discussion}

It was reported that nearly $25 \%$ of patients with newly diagnosed epilepsy are older. ${ }^{27,28}$ However, the knowledge about surgical treatment for drug-resistant epilepsy in older patients is limited. Herein, we retrospectively reported the seizure outcomes of a case series including 45 patients aged 45 years or older who had undergone resective surgery for drug-resistant TLE. We then analyzed the prognostic factors for postoperative seizure outcomes. To the best of our knowledge, this is the first study to analyze the prognostic factors of postoperative seizure outcomes in older patients with TLE.

An increasing number of studies have suggested that resective surgery for drug-resistant epilepsy in elderly patients is safe and effective. $1,7,8,22,24-26$ However, contradictory findings have also been reported. ${ }^{13,29}$ Similar controversy also exists regarding surgical treatment for TLE in older patients. Grivas et al. ${ }^{13}$ and Murphy et al. ${ }^{24}$ found that the seizure outcomes of resective surgery for TLE in older patients are similar to those in younger patients. However, Sirven et al. ${ }^{29}$ reported contradictory results. Some studies have also shown that the seizure-free rates of resective surgical treatment among elderly TLE patients are heterogeneous, ranging from $30 \%$ to $81 \% .^{8,13,22,24,29}$ These wide-ranging postoperative seizure outcomes may be attributable to differences in etiologies or issues associated with small sample sizes. ${ }^{24,25}$ In the present study, we found that $73.3 \%$ of older TLE patients were seizure free postoperatively, over a mean follow-up of 4.5 years. This finding was similar to the results observed in other large series on surgical treatment for TLE that included both younger and older patients. ${ }^{11,15,17}$

The notion that the surgical risk may increase in older patients is another factor that limits the use of resective surgery for drug-resistant epilepsy in such patients. ${ }^{9}$ In the literature, the rate of temporary surgical complications in older TLE patients ranges from $7.7 \%$ to $25 \%$, and the rate of permanent surgical complications ranges from $0.0 \%$ to $3.8 \% .8,13,22,24,29$ Murphy et al. ${ }^{24}$ has reported a comparable rate of surgical complications in younger and older patients, whereas Grivas et al..$^{13}$ observed a higher risk of complications in older patients. In the present study, temporary surgical complications were observed in $13.3 \%$ of patients, whereas only $2.2 \%$ of patients had permanent surgical complications. These results are similar to those following surgical treatment for TLE in a younger patient cohort. ${ }^{5,13,24}$ In addition, no wound infections or postoperative intracerebral hemorrhages were observed in the present study, even though $17.8 \%$ of patients had preoperative comorbidities such as hypertension and/or diabetes.

It is imperative that patients who have undergone epilepsy surgery are appropriately selected. Many studies have assessed the prognostic factors of surgical treatment for TLE; however, little is known about these prognostic factors in older patients. In the present study, we found that MRI-detected epileptogenic lesions are the only independent predictor of a favorable seizure outcome in older patients with TLE who have undergone resective surgery. Various studies have shown MRI-detected epileptogenic lesions to be a predictor of favorable seizure outcomes in patients undergoing surgical treatment for TLE. $3,4,6,12,21,30$ In the present study, we demonstrated that such MRI-detected lesions are the only independent predictor of favorable seizure outcomes in surgically treated older patients with TLE. This finding may be attributable to the histopathology of patients with MRI-negative findings. Among the 6 patients with MRI-negative findings, the histopathology was FCD type I in 5 and nonspecific gliosis in 1 patient, both of which are considered diffuse pathology and are difficult to resect completely. ${ }^{21}$

Another often-cited reason to withhold resective surgery in older patients with drug-resistant epilepsy is their relatively longer seizure duration..$^{15,29}$ A longer history of seizures can lead to the formation of secondary epileptogenic zones and thus result in the failure of epilepsy surgery. ${ }^{15,23}$ However, we did not find such associations in the present study. Other factors such as age at seizure onset, age at surgery, and seizure frequency ${ }^{2,5,16,29}$ have been re- 
ported as independent predictors of seizure outcomes, but we did not find similar results. This finding may be attributable to the character of the cases in the present study.

\section{Study Limitations}

There are some limitations to the present study. First, data were collected retrospectively, and the inherent biases associated with the retrospective nature of this study cannot be avoided. Second, the sample size was relatively small. A large clinical sample is needed to establish clearer conclusions in the future. Third, we failed to evaluate the influence of surgery on patients' quality of life and neuropsychological outcomes, which are important aspects of surgical decision-making. Despite these limitations, the present study provides useful information on resective surgical treatment for older patients with TLE.

\section{Conclusions}

Data from the present study indicated that resective surgery is a safe and effective treatment for older patients with drug-resistant TLE. An MRI-negative finding is the only independent predictor of unfavorable seizure outcomes.

\section{Acknowledgments}

This work was supported by grants from the National Natural Science Foundation of China (81790654 and 81790650 to G.L.).

\section{References}

1. Acosta I, Vale F, Tatum WO IV, Benbadis SR: Epilepsy surgery after age 60. Epilepsy Behav 12:324-325, 2008

2. Aguglia U, Beghi E, Labate A, Condino F, Cianci V, Mumoli L, et al: Age at onset predicts good seizure outcome in sporadic non-lesional and mesial temporal sclerosis based temporal lobe epilepsy. J Neurol Neurosurg Psychiatry 82:555-559, 2011

3. Antel SB, Li LM, Cendes F, Collins DL, Kearney RE, Shinghal R, et al: Predicting surgical outcome in temporal lobe epilepsy patients using MRI and MRSI. Neurology 58:15051512,2002

4. Berkovic SF, McIntosh AM, Kalnins RM, Jackson GD, Fabinyi GC, Brazenor GA, et al: Preoperative MRI predicts outcome of temporal lobectomy: an actuarial analysis. Neurology 45:1358-1363, 1995

5. Choi H, Sell RL, Lenert L, Muennig P, Goodman RR, Gilliam FG, et al: Epilepsy surgery for pharmacoresistant temporal lobe epilepsy: a decision analysis. JAMA 300:24972505,2008

6. Clusmann H: Predictors, procedures, and perspective for temporal lobe epilepsy surgery. Semin Ultrasound CT MR 29:60-70, 2008

7. Costello DJ, Shields DC, Cash SS, Eskandar EN, Cosgrove GR, Cole AJ: Consideration of epilepsy surgery in adults should be independent of age. Clin Neurol Neurosurg 111:240-245, 2009

8. Dewar S, Eliashiv D, Walshaw PD, Engel J Jr, Fried I, Moseley BD: Safety, efficacy, and life satisfaction following epilepsy surgery in patients aged 60 years and older. J Neurosurg 124:945-951, 2016

9. Engel J Jr, Shewmon DA: Overview: who should be considered a surgical candidate? In Engel J, Jr (ed): Surgical Treatment of the Epilepsies, ed 2. Philadelphia: Raven Press, 1993, pp 23-34
10. Engel J Jr, Wiebe S, French J, Sperling M, Williamson P, Spencer D, et al: Practice parameter: temporal lobe and localized neocortical resections for epilepsy. Epilepsia 44:741-751, 2003

11. Englot DJ, Rolston JD, Wang DD, Sun PP, Chang EF, Auguste KI: Seizure outcomes after temporal lobectomy in pediatric patients. J Neurosurg Pediatr 12:134-141, 2013

12. Gilliam F, Bowling S, Bilir E, Thomas J, Faught E, Morawetz $\mathrm{R}$, et al: Association of combined MRI, interictal EEG, and ictal EEG results with outcome and pathology after temporal lobectomy. Epilepsia 38:1315-1320, 1997

13. Grivas A, Schramm J, Kral T, von Lehe M, Helmstaedter C, Elger CE, et al: Surgical treatment for refractory temporal lobe epilepsy in the elderly: seizure outcome and neuropsychological sequels compared with a younger cohort. Epilepsia 47:1364-1372, 2006

14. Hu WH, Zhang C, Zhang K, Meng FG, Chen N, Zhang JG: Selective amygdalohippocampectomy versus anterior temporal lobectomy in the management of mesial temporal lobe epilepsy: a meta-analysis of comparative studies. J Neurosurg 119:1089-1097, 2013

15. Janszky J, Janszky I, Schulz R, Hoppe M, Behne F, Pannek HW, et al: Temporal lobe epilepsy with hippocampal sclerosis: predictors for long-term surgical outcome. Brain 128:395-404, 2005

16. Jeong SW, Lee SK, Kim KK, Kim H, Kim JY, Chung CK: Prognostic factors in anterior temporal lobe resections for mesial temporal lobe epilepsy: multivariate analysis. Epilepsia 40:1735-1739, 1999

17. Jobst BC, Cascino GD: Resective epilepsy surgery for drugresistant focal epilepsy: a review. JAMA 313:285-293, 2015

18. Josephson CB, Dykeman J, Fiest KM, Liu X, Sadler RM, Jette N, et al: Systematic review and meta-analysis of standard vs selective temporal lobe epilepsy surgery. Neurology 80:1669-1676, 2013

19. Kuang Y, Yang T, Gu J, Kong B, Cheng L: Comparison of therapeutic effects between selective amygdalohippocampectomy and anterior temporal lobectomy for the treatment of temporal lobe epilepsy: a meta-analysis. Br J Neurosurg 28:374-377, 2014

20. Kwan P, Arzimanoglou A, Berg AT, Brodie MJ, Hauser WA, Mathern G, et al: Definition of drug resistant epilepsy: consensus proposal by the ad hoc Task Force of the ILAE Commission on Therapeutic Strategies. Epilepsia 51:1069-1077, 2010

21. McIntosh AM, Kalnins RM, Mitchell LA, Fabinyi GC, Briellmann RS, Berkovic SF: Temporal lobectomy: long-term seizure outcome, late recurrence and risks for seizure recurrence. Brain 127:2018-2030, 2004

22. McLachlan RS, Chovaz CJ, Blume WT, Girvin JP: Temporal lobectomy for intractable epilepsy in patients over age 45 years. Neurology 42:662-665, 1992

23. Morrell F: Secondary epileptogenesis in man. Arch Neurol 42:318-335, 1985

24. Murphy M, Smith PD, Wood M, Bowden S, O'Brien TJ, Bulluss KJ, et al: Surgery for temporal lobe epilepsy associated with mesial temporal sclerosis in the older patient: a longterm follow-up. Epilepsia 51:1024-1029, 2010

25. O’Dwyer R, Byrne R, Lynn F, Nazari P, Stoub T, Smith MC, et al: Age is but a number when considering epilepsy surgery in older adults. Epilepsy Behav 91:9-12, 2019

26. Punia V, Abdelkader A, Busch RM, Gonzalez-Martinez J, Bingaman W, Najm I, et al: Time to push the age limit: epilepsy surgery in patients 60 years or older. Epilepsia Open 3:73-80, 2018

27. Ramsay RE, Rowan AJ, Pryor FM: Special considerations in treating the elderly patient with epilepsy. Neurology 62 (5 Suppl 2):S24-S29, 2004

28. Sander JW, Hart YM, Johnson AL, Shorvon SD: National 
General Practice Study of Epilepsy: newly diagnosed epileptic seizures in a general population. Lancet 336:1267-1271, 1990

29. Sirven JI, Malamut BL, O'Connor MJ, Sperling MR: Temporal lobectomy outcome in older versus younger adults. Neurology 54:2166-2170, 2000

30. Sun Z, Zuo H, Yuan D, Sun Y, Zhang K, Cui Z, et al: Predictors of prognosis in patients with temporal lobe epilepsy after anterior temporal lobectomy. Exp Ther Med 10:1896-1902, 2015

31. Wieser HG, Blume WT, Fish D, Goldensohn E, Hufnagel A, King D, et al: ILAE Commission Report. Proposal for a new classification of outcome with respect to epileptic seizures following epilepsy surgery. Epilepsia 42:282-286, 2001

\section{Disclosures}

The authors report no conflict of interest concerning the materials or methods used in this study or the findings specified in this paper.

\section{Author Contributions}

Conception and design: Luan, He. Acquisition of data: He. Analysis and interpretation of data: He. Drafting the article: He. Critically revising the article: Luan, Zhou, Li. Reviewed submitted version of manuscript: all authors. Approved the final version of the manuscript on behalf of all authors: Luan. Statistical analysis: He. Administrative/technical/material support: Zhou, Guan, Zhai. Study supervision: Zhou, Guan, Zhai, Li.

\section{Correspondence}

Guoming Luan: Capital Medical University, Beijing, China. luangm_3@163.com. 